\title{
APPLICATIONS OF SCANNING TUNNELING MICROSCOPY TO SOLID STATE PHYSICS
}

\author{
H. van Kempen, E.J.G. Boon, M.C.M.M. van DeR WIelen, \\ J.W.G. WILdöER, M.W.J. Prins, R. JANSEN and R. SCHAD \\ Research Institute for Materials, University of Nijmegen \\ Toernooiveld 1, 6525 ED Nijmegen, The Netherlands
}

Due to the dependence of the tunnel current to material properties like work function, density of states, and spin polarization the scanning tunneling microscope can be used to study a number of solid state physics problems. This will be illustrated with some examples. The presented examples have in common that also the role of the tip properties have to be taken explicitly into account.

PACS numbers: 61.16.Ch, 68.35.Dv, 71.55.Eq

\section{Introduction}

As is well known, the scanning tunneling microscope (STM) has excellent imaging capabilities, which have allowed near routine observation of atomic positions at surfaces. This capability is based on the extreme sensitivity of the tunnel current on a tip-sample distance. However, the tunnel current depends also on material properties like work function, density of states, and spin polarization of the conduction electrons. This dependence has given rise to a number of spectroscopic techniques with which solid state physics research can be carried out on a very small spatial scale. Of course one has to keep in mind that the tunnel current is not only dependent on the sample properties but also an important role might be played by the tip.

In this paper some examples will be given where the role of the tip cannot be neglected, and where it is in some cases even a factor of major importance. The experiments involve a study of dopants in semiconductors, an observation of a single trapped electron, and a novel near optical field method for the observation of magnetic domains.

\section{Dopants in GaAs(110)}

Dopants in semiconductors determine the most important properties of semiconductors and semiconducting devices. The direct observation of dopants allows us to determine their spatial distribution in semiconductors but also to study the 
electronic structure in their environment. For the first aspect, room temperature experiments are suitable (see for example Refs. [1] and [2]). However, for the study of the electronic structures low temperature experiments are preferred to get higher energy resolution.

In this section we report the direct imaging of electrically active Si-dopants near the GaAs(110) surface with a scanning tunneling microscope at a temperature of $4.2 \mathrm{~K}$. Clean surfaces are obtained by in situ cleavage of a (001) oriented wafer at liquid helium temperature. As a result of the low temperature the freshly cleaved surface is only exposed to a very clean helium atmosphere where, for example, the partial oxygen pressure is lower than $10^{-17}$ torr. This allows nearly unlimited observation time without contamination of the sample. The experiments are performed in low-temperature STMs, which are fully described by Wildöer et al. [3] and by Dubois et al. [4].

STM measurements on the GaAs(110) surface have been performed at $4.2 \mathrm{~K}$. Figure 1a shows an empty state image of the $\mathrm{GaAs}(110)$ surface. The scan area is $31 \mathrm{~nm} \times 31 \mathrm{~nm}$. Clearly two hillock features are visible in the surface topography. These features are superimposed on the gallium sublattice and are induced by ionised silicon donor atoms positioned below the surface, see Ref. [1]. The positive charge of the doping atom locally increases the density of states and causes the tip to retract. In Fig. 1b a filled state image is shown of exactly the same surface area. The hillock features are replaced by two ring like structures superimposed on the As sublattice. An averaged cross section through one of the features is shown in Fig. 1c. The atomic lattice is filtered out in this cross section. Two maxima and one minimum are visible. The amplitude of the corrugation decays with increasing distance from the centre.

The difference between Figs. 1 $a$ and $b$ can be explained by the effect of tip induced band bending. Due to the limited amount of free carriers, the electric field between tip and sample penetrates into the semiconductor material. At a positive sample voltage (empty state image), the electric field will push the electrons away from the surface. A depletion region is created in which all doping atoms are ionised. It is the Coulomb potential which induces the hillock features in Fig. 1a. At a negative sample voltage (filled state image), electrons are pulled towards the surface. An accumulation layer is formed and doping atoms are no longer ionised. The extra charge of the donor atom is screened by free charge carriers. The ring like structures are caused by the screening.

In fact the ring like structures are oscillations in the surface state density. They exist when the screened potential is of the order of the electron wavelength. These kinds of oscillations in the charge density are known as Friedel oscillations. The oscillation period is equal to $\lambda_{\mathrm{F}} / 2$ for $r \rightarrow \infty$, with $\lambda_{\mathrm{F}}$ the electron Fermi wavelength and $r$ the distance to the centre of the point charge. In our STM images, we can only measure Friedel oscillations at a short distance from the point charge. Therefore, we can only use the expression for the oscillation period as a first order approximation. From Fig. 1c we derive a value of about $10 \mathrm{~nm}$ for the electron Fermi wavelength. Following the calculations in Refs. [5] and [6] we find a value of $0.28 \mathrm{eV}$ for the tip induced surface potential. Using the dispersion relation for free electrons, $E_{\mathrm{F}}=\hbar^{2} k_{\mathrm{F}}^{2} / 2 m^{*}$ (with $k_{\mathrm{F}}=2 \pi / \lambda_{\mathrm{F}}$ the electron Fermi wave 

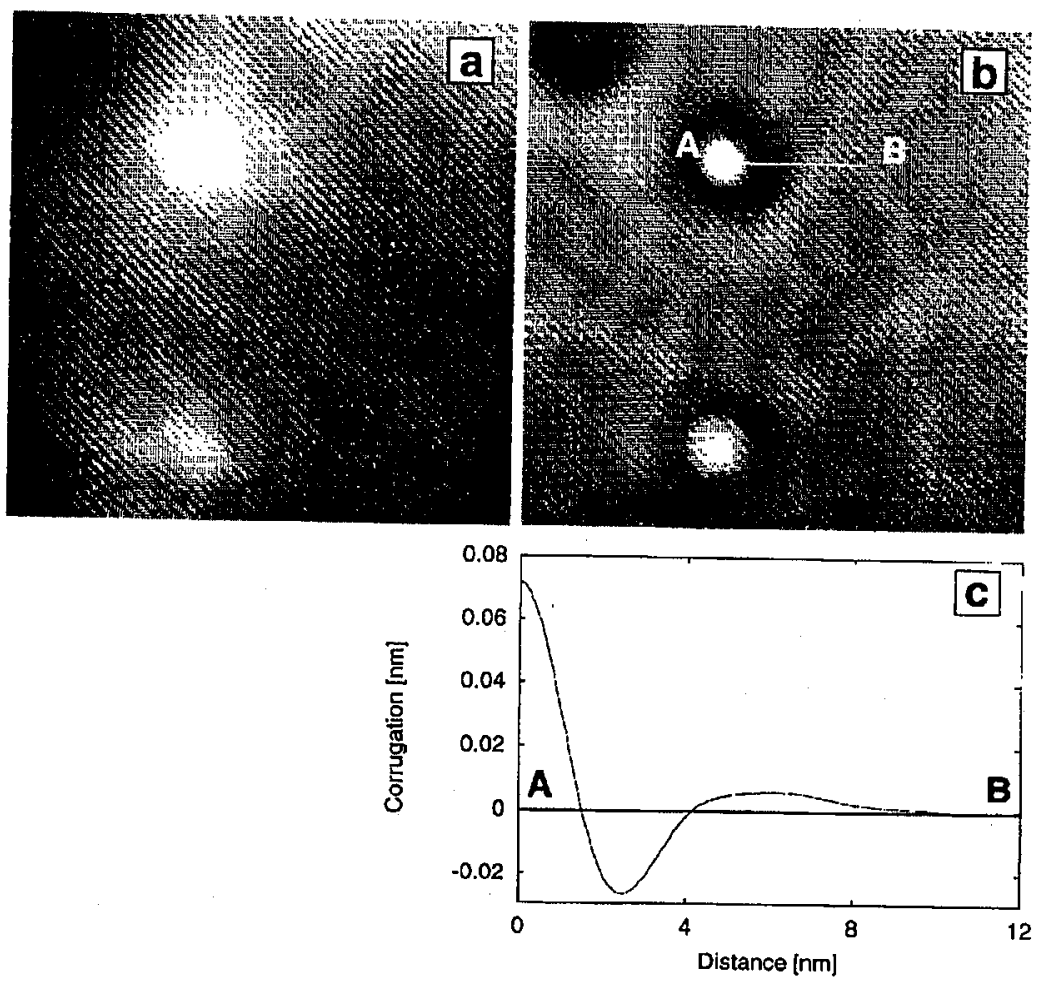

Fig. 1. Figures (a) and (b) show two STM images on the (110) plane of a $6 \times 10^{17} \mathrm{~cm}^{-3}$ Si-doped GaAs sample. Both images are scans from the same surface area, measured at different bias polarity. Scan size: $31 \mathrm{~nm} \times 31 \mathrm{~nm}$. Set-point current is $20 \mathrm{pA}$. (a) Empty state image measured at $+1.0 \mathrm{~V}$ sample voltage. Grey scale range: 0 to $0.29 \mathrm{~nm}$. Two hillock features are visible superimposed on the gallium sublattice. (b) Filled state image measured at $-2.1 \mathrm{~V}$ sample voltage. Grey scale range: 0 to $0.16 \mathrm{~nm}$. At the location of the hillock features in (a), ring like structures are visible superimposed on the arsenide sublattice. A cross section through one of these structures along line $A-B$ is shown in (c). The atomic lattice is filtered out.

vector, $m^{*}=0.067 m_{0}$ the effective electron mass), we calculate a value of $9 \mathrm{~nm}$ for $\lambda_{\mathrm{F}}$. This is in surprisingly good agreement with the measured electron Fermi wavelength.

Next to Si-doped samples also Zn-doped samples have been studied. The substitutional $\mathrm{Zn}_{\mathrm{Ga}}$-atom has two valence electrons and therefore is not donating but accepting an electron. Again, screening effects are expected around the negative charged Zn-atoms by free charge carriers (holes). Figure 2 shows an empty state image of such a $\mathrm{Zn}$-doped sample with a doping concentration $>8 \times 10^{18} \mathrm{~cm}^{-3}$. The picture is measured at a sample voltage of $+1.8 \mathrm{~V}$. The bright features in the image are induced by the negative charged dopants. These features are not circular symmetric but have a triangular shape. All features have the same orien- 


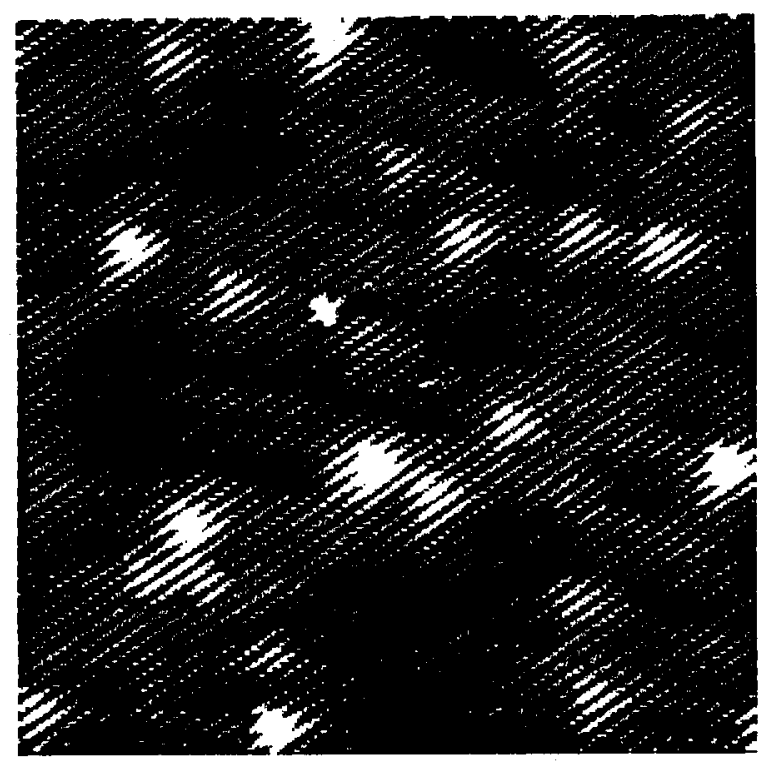

Fig. 2. Empty state image on the (110) plane of a Zn-doped GaAs sample. Sample voltage: $+1.8 \mathrm{~V}$, tunnel current: $200 \mathrm{pA}$. Dopant induced features have a triangular shape.

tation. The same kind of features has been observed before in room-temperature measurements by Zheng et al. [7]. We do not have an explanation for this effect yet. At a lower doped sample $\left(5 \times 10^{17} \mathrm{~cm}^{-3}\right)$, we did observe the screening effects we expected, similar to the effects around Si-dopants, see Ref. [8].

\section{Single electron box}

A series connection of two tunnel junctions with small capacitances can give rise to steps in the $I-V$ characteristics. This so-called "Coulomb Staircase" is a result of the charging effects of the junctions and becomes apparent when the Coulomb charging energy of a single electron becomes larger than the thermal energy: $e^{2} /(2 C) \gg 2 k T[9,10]$. An interesting arrangement to observe the Coulomb Staircase is to use a small metallic cluster as the central electrode of the double junction while one junction is formed by depositing the cluster on a metal substrate covered by a thin insulating layer. The other junction is formed by the STM tip placed above the cluster (e.g. Ref. [11]). With this configuration the capacitances are small enough to observe charging effects at liquid helium temperature and even at room temperature $[12,13]$. An added advantage for fundamental studies is that the tip-cluster junction can be varied by moving the tip.

When one of the junctions is replaced by a small capacitor, the tunnel current cannot flow any longer. But still charging of the central electrode with a single electron can take place above a certain voltage threshold $V_{\mathrm{c}}$

$$
V_{\mathrm{c}}=-e /\left(2 C_{\mathrm{T}}\right)
$$


where $C_{\mathrm{T}}$ is the capacitance of the tunnel junction. (This is a simplified expression, neglecting work function differences of the electrodes and a possible charge offset of the central electrode. The full expression can be found in Ref. [14] and a further discussion in Ref. [15].)

The arrangement we use, is indicated in Fig. 3. The tip-grain distance is too large to allow tunneling except when the grain is observed by the STM. So the grain can act as a single electron box. During scanning, the tip enters and exits the region where the capacitance is above the critical value and the cluster will be charged and discharged accordingly.

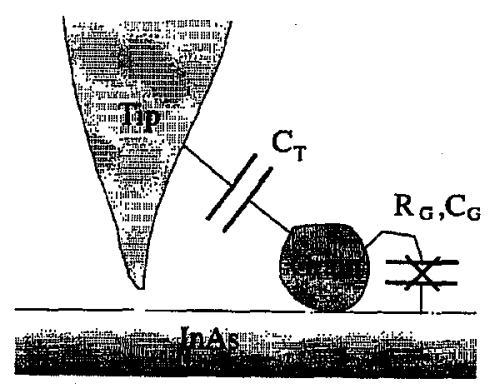

Fig. 3. Principles of single electron box formed by STM tip, conducting grain and substrate. Assumed is the presence of a tunnel barrier between grain and substrate.
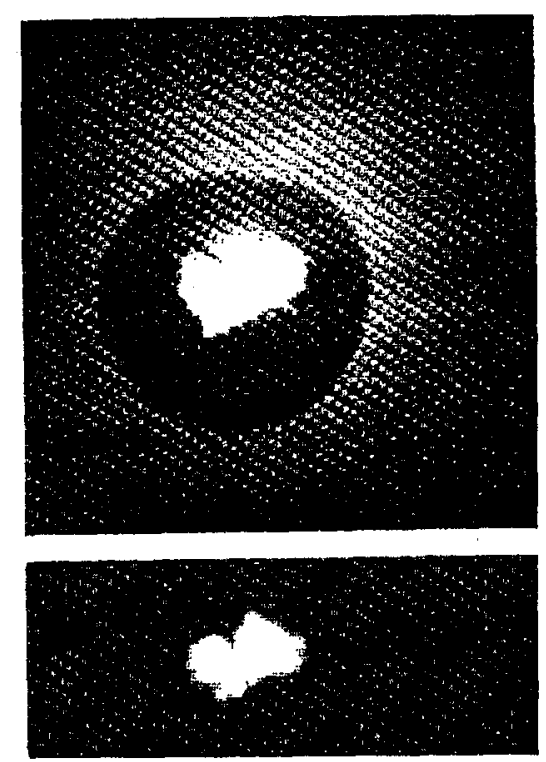

Fig. 4. Constant-current images at sample voltages of (upper panel) $-400 \mathrm{mV}$ and (lower panel) $400 \mathrm{mV}$. The scan is $230 \AA^{2}$. The tunnel current is $150 \mathrm{pA}$. 
Generally, it is difficult to study a single electron box because no current flows in the leads and so the charging effects can only be observed by some way sensing the charge of the central electrode directly [16]. In our case however, the InAs substrate acts as a detector. The charging of the grain causes a jump in the band bending which shows up in the images of the InAs (Fig. 4). During this measurement the applied voltage is constant, so according to Eq. (1), the tip-grain capacitance determines the charging. The jump in band bending manifests itself as a change in tunnel current. This is visible in Fig. 4 as a roundish boundary between two regions. The boundary is a line of equal capacitance, corresponding to the critical value $V_{c}$ for charging the grain. So the shape of this roundish line indicates a convolution of tip and cluster shape. As is obvious, a different voltage will give a different critical capacitance. This has been indeed observed [15].

In a different perspective, the metal cluster can be considered as a nanoscopic gate, whose charge variation with discrete electron charges results in discrete variations of the semiconductor band bending. Such small gates are considered as candidates for parts of single electron memory devices.

\section{Magneto-optical near-field microscopy}

Magnetic imaging is an important issue in both fundamental science and modern technology. Further development of magnetic data-storage technology requires magnetic imaging with a resolution better than the size of the magnetic structures. As the bit size in magnetic recording goes beyond the optical diffraction limit, the classical imaging techniques have reached their limits. We developed a microscope combining the classical magneto-optical contrast mechanism with an STM operating in the optical near-field: the magneto-optical near-field scanning tunneling microscope, short MONF-STM. The advantages of this microscope, besides the sub-micrometer magnetic resolution and the atomic topography resolution, are the absence of magnetic tip-sample interaction, the fact that it can be operated in external magnetic fields and under ambient conditions.

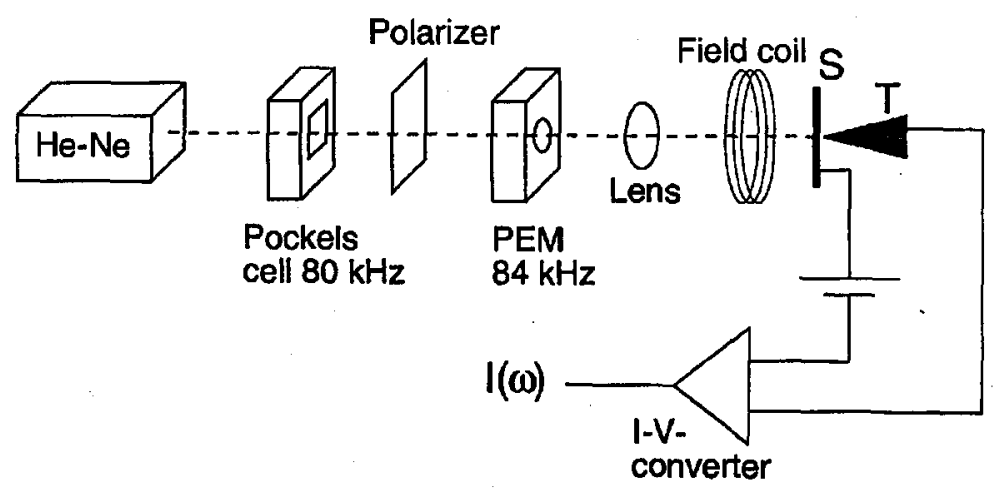

Fig. 5. Experimental arrangement of MONF-STM. See the text for further explanation. 
Essentially the microscope consists of two parts (Fig. 5): first, the illumination of the magnetic sample with circularly polarised laser light (20 micrometer beam diameter) which passes through the magnetic domains and experiences an intensity attenuation depending on the direction of the magnetization, an effect called magnetic circular dichroism (MCD). This way the light intensity distribution behind the sample reflects the magnetic information of the sample. Second, the light intensity after transmission through the sample is detected locally using a photosensitive semiconducting GaAs tip. The amount of electron-hole pairs which are generated in the tip by the light is proportional to the intensity of the incoming light. These photo-electrons move in the electric field of the band bending towards the apex of the tip producing a tunnel current even at zero bias voltage [17-19]. This way, the tunnel current contains both the magnetic information and the topography. The normal feedback loop of the STM keeps the tunnel current constant and reproduces this way the topography of the sample surface with a very high accuracy. The magnetic information is filtered out by switching the polarization of the incoming laser light between left and right handed circular polarization with a frequency of $84 \mathrm{kHz}$. The frequency of this helicity modulation is faster than the bandwidth of the feedback loop so that the magnetic information is contained in the tunnel current as amplitude modulation and can be detected frequency selective using a lock-in amplifier. A small intensity modulation of a few percent at a frequency of $80 \mathrm{kHz}$ is also imposed (see Fig. 5). The response of the tunnel current to this modulation is detected by a second lock-in amplifier and serves as a reference signal to normalize the MCD signal.

The GaAs tips ( $p$-type of $10^{17} \mathrm{~cm}^{-3}$ doping density) were prepared by cleaving (001) wafers along (110) and (1-10) directions, forming a corner bounded by these planes. The cleavage produced well-defined corners with a tip apex radius of $100 \mathrm{~nm}$ suitable for atomic resolution on graphite. As a test sample served a Co/Pt superlattice with perpendicular magnetization consisting of a $6 \AA \mathrm{Pt}$ base layer and 20 pairs of $3.5 \AA \mathrm{Co} / 6 \AA \mathrm{Pt}$ layers evaporated on a glass substrate. The sample was homogeneously magnetised except for rows of thermomagnetically written bits of opposite magnetization with micrometer dimension [20].

Figure 6 demonstrates the magneto-optical imaging capabilities of our semiconductor tips. It shows a $2 \times 7 \mu \mathrm{m}$ image of the magnetic bits, taken in 8 minutes time. The bits were detected by the CD signal measured while scanning. Indeed, we observe that the inter-bit distance is approximately $2 \mu \mathrm{m}$. Outside the area with the bits a CD signal with a uniform sign has been observed, whereas on the bits a sign reversal of this signal was found. The observation of the sign reversal is in agreement with the fact that the bits have opposite magnetization with respect to the rest of the sample.

Since both the beam diameter of the laser light and the tip dimensions are macroscopic the magnetic resolution is determined by other parameters. This resolution is given by the active detector size of the tip, represented by the so-called photocarrier collection radius, which is essentially determined by the following parameters:

- the optical penetration depth of the light, 
- the minority carrier diffusion length,

- the profile of the band-bending region.

All of these can be tuned to be of sub-wavelength extent. The main optical parameter is the penetration depth of the light, which is $250 \mathrm{~nm}$ and $130 \mathrm{~nm}$ in GaAs for light wavelengths of $633 \mathrm{~nm}$ and $532 \mathrm{~nm}$, respectively. This was used to improve the resolution from $250 \mathrm{~nm}$ to about $100 \mathrm{~nm}$ when going from red (used for obtaining Fig. 6) to green laser light for a p-type GaAs tip of $10^{17} \mathrm{~cm}^{-3}$ doping density [21].

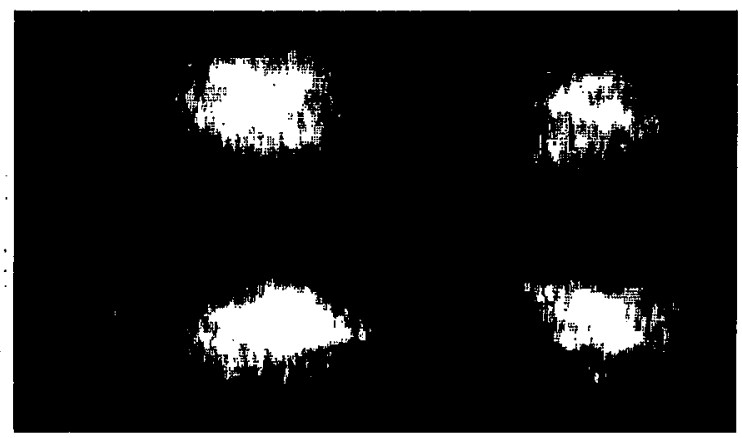

Fig. 6. Circular dichroism image of magnetic bits prewritten in a Pt/Co multilayer. See the text for further details.

A measurement scheme that is closely related to the above presented technique is spin-polarised tunneling by optical spin-orientation in GaAs [22]. The MCD is sensitive to the bulk magnetization, whereas spin-polarised tunneling is sensitive to the spin-polarization of electronic states at the sample surface. The respective effects may be separated by their dependence on excitation wavelength, surface preparation, and bias voltage. Ideally, one would like to combine the two measurements, so as to be able to simultaneously measure sample topography, bulk magnetization, and surface spin-structure with (sub-)nanometer resolution.

\section{Acknowledgment}

This work was supported by the Stichting Fundamenteel Onderzoek der Materie (FOM), which is financially supported by the Nederlandse Organisatie voor Wetenschappelijk Onderzoek (NWO). We also like to acknowledge the support of the Brite-Euram Programme of the European Community (contract number BRE2-CT93-0569). We thank Dr. H.W. van Kesteren, Philips Research Laboratory, for supplying the CoPt-sample.

\section{References}

[1] J.F. Zheng, X. Liu, N. Newman, E.R. Weber, D.F. Ogletree, M. Salmeron, Phys. Rev. Lett. 72, 1490 (1994).

[2] M.B. Johnson, O. Albrektsen, R.M. Feenstra, H.W.M. Salemink, Appl. Phys. Lett. 63, 2923 (1993). 
[3] J.W.G. Wildöer, A.J.A. van Roij, H. van Kempen, C.J.P.M. Harmans, Rev. Sci. Instrum. 65, 2849 (1994).

[4] J.G.A. Dubois, J.W. Gerritsen, J.G.H. Hermsen, H. van Kempen, Rev. Sci. Instrum. 66, 4146 (1995).

[5] R.M. Feenstra, A. Stroscio, J. Vac. Sci. Technol. B 5, 923 (1987).

[6] M.C.M.M. van der Wielen, A.J.A. van Roij, H. van Kempen, Phys. Rev. Lett. 76, 1075 (1996).

[7] Z.F. Zheng, M.B. Salmeron, E.R. Weber, Appl. Phys. Lett. 64, 1836 (1994).

[8] M.C.M.M. van der Wielen, A.J.A. van Roij, H. van Kempen, to be published.

[9] H.R. Zeller, I. Giaever, Phys. Rev. 181, 789 (1969).

[10] K.K. Likharev, IBM J. Res. Develop. 32, 144 (1988).

[11] P.J.M. van Bentum, R.T.M. Smokers, H. van Kempen, Phys. Rev. Lett. 60, 2543 (1988).

[12] H. Nejoh, Nature 353, 6345 (1991).

[13] C. Schönenberger, H. van Houten, H.C. Donkersloot, Europhys. Lett. 20, 249 (1992).

[14] C.T. Black, M.T. Tuominen, M. Tinkham, Phys. Rev. B 50, 7888 (1994).

[15] J.W.G. Wildoër, A.J.A. van Roij, C.J.P.M. Harmans, H. van Kempen, Phys. Rev. $B$ 53, 10695 (1996).

[16] P. Lafarge, H. Potier, E.R. Williams, D. Esteve, C. Urbina, M.H. Devoret, $Z$. Phys. B 85, 327 (1991).

[17] M.W.J. Prins, M.C.M.M. van der Wielen, R. Jansen, D.L. Abraham, H. van Kempen, Appl. Phys. Lett. 64, 1207 (1994).

[18] M.W.J. Prins, A.P. van Gelder, Physica B 218, 297 (1996).

[19] M.W.J. Prins, R. Jansen, R.H.M. Groeneveld, A.P. van Gelder, H. van Kempen, Phys. Rev. B 53, 8090 (1996).

[20] M.W.J. Prins, R.H.M. Groeneveld, D.L. Abraham, H. van Kempen, Appl. Phys. Lett. 66, 1141 (1995).

[21] M.J.P. Stoelinga, M. Sc. Thesis, University of Nijmegen, Nijmegen 1996.

[22] M.W.J. Prins, R. Jansen, H. van Kempen, Phys. Rev. B 53, 8105 (1996). 